\title{
INVESTIGATIONS ON THE DYNAMIC STATION KEEPING OF AN UNDERACTUATED AUTONOMOUS UNDERWATER ROBOT
}

\author{
Santhakumar, M. \& Asokan, T. \\ Robotics Research Laboratory, Department of Engineering Design, \\ Indian Institute of Technology Madras, Chennai 600036, India \\ E-Mail: santharadha@gmail.com
}

\begin{abstract}
In this paper, a new method for station keeping of underactuated underwater robots in the presence of underwater currents and external disturbances is proposed. Three small additional thrusters are introduced for station keeping purpose which are less power consuming and are actuated only during the station keeping mode. These thrusters are located in such a way that the generated forces and torque are enough to compensate the underwater currents and disturbances. Station keeping thrusters' locations and directions are nearly optimized using Taguchi's robust design method. The effect of additional thrusters on robot tracking control performance is investigated and the results are presented. The effectiveness of the proposed configuration is demonstrated with the help of hardware-in-the-loop (HIL) simulations using an experimental autonomous underwater robot. The underwater current effect on the corresponding motions of the underwater robot is investigated and some interesting phenomena with respect to different underwater current amplitudes and directions are observed. Robustness of the proposed configuration is also investigated.

(Received in December 2010, accepted in April 2011. This paper was with the authors 1 month for 2 revisions.)
\end{abstract}

Key Words: Station Keeping, Underwater Robot, Underwater Current, Tracking Control, Underactuated Control

\section{INTRODUCTION}

In the modern years, a great amount of research has been accomplished regarding the operation of autonomous underwater robots (AURs) and they are playing a crucial role in exploration and utilization of resources located at deep oceanic environments. They are found to be very essential to many underwater missions (which are risky in general) such as oceanographic observations, bathymetric surveys, ocean floor analysis, military applications, recovery of lost man-made objects, etc. [1,2]. The ability of an underwater robot to remain in its position / station is critical for the success of many underwater missions. The robot needs to maintain or keep its geometric body centre at a given coordinate position. This is slightly complex and difficult due to coupled, nonlinear robot dynamics with uncertain hydrodynamic parameters [3,4]. Moreover, AURs present a challenging control problem since most of them are underactuated, i.e., they have fewer actuated inputs than degrees of freedom (DOF), imposing non-integrable acceleration constraints making control design a hard task $[2,5]$. Underwater robot control is mainly divided into two major categories such as tracking and set-point control. Many researchers have approached the first one (tracking) and many solutions have been proposed in the literature, with varying degrees of success, which are summarized in [5-7]. Tracking control techniques proposed in literature can be broadly classified into two major categories: adaptive control and robust control $[2,8]$. In adaptive control the controller parameters are automatically varied to maintain a satisfactory level of 
performance when the system parameters are unknown and / or time varying. Robust control refers to the control of uncertain plants with unknown disturbance signals, uncertain dynamics and imprecisely known parameters making use of special fixed controllers. Underactuated underwater robot tracking control problem is approached in many different methods. In these studies, most of them have used Lyapunov based back-stepping approach [9-11]. Among these, adaptive control is considered to be better for plant uncertainty. However, it is computationally intensive for higher order systems and requires exact knowledge of the dynamic parameters, apart from the computation of inverse Jacobian matrix. The robust control scheme provides a satisfactory performance with a simple control structure, but comes with undesired high control activity at steady state. On the other hand, the commonly used proportional-integral-derivative (PID) control $[6,12]$ does not require any information of the plant dynamics and has a simple standard structure. Moreover, owing to modelling uncertainties a more sophisticated control scheme is not necessarily more efficient than a well-tuned PID controller.

However, only limited attempts have been made in the field of dynamic station keeping control of underwater vehicles [13-18] and most of the available literatures on station keeping focus on the visual sensing and observer design aspects. Brutzman et al. [13] have analysed the near surface manoeuvring and station keeping in the presence of wave effects. However, in this work the vehicle has considered as fully actuated and moreover this work focused towards development of simulation tool. Riedel [17] has proposed disturbance compensation controller based on extended Kalman filter (EKF) in the shallow water region, which provides control commands based on estimation of disturbances (wave effects). There was no formal proof for stability is available. There are few attempts made towards station keeping control based on visual sensing [16-21]. In these studies, visual sensing has considered as the core and focussed towards that. However, the controller design and the actuators characteristics of the vehicle are assumed to be an ideal case. In addition, robots are considered as fully actuated and same tracking actuators are used for the station keeping purpose. Very few attempts can be found in the area of underactuated underwater robot station keeping [14, 22]. Koh et al. [14] have approached the station keeping problem with available thrusters configuration using proportional-derivative (PD) control for remotely operated vehicle. However, in this study the roll and the pitch angles are considered as unactuated states. Previous studies limitations of are as follows:

- Underwater vehicle is considered as fully actuated,

- Same actuators which are used for tracking control are used for station keeping, therefore consumption of power is high and controller design is complex in nature and,

- Limited numbers of attempts are considered underwater currents and their effects.

Santhakumar and Asokan [22] have considered additional thrusters are available which are for only station keeping purpose and vehicle performance is investigated on the horizontal plane through numerical simulations. However, in this study the effect of these additional thrusters inclusion for the vehicle tracking performance is not analysed. Therefore in this work, our previous study is extended to three dimensional space and investigates the vehicle station keeping performances under different operating conditions.

In this paper, it focuses on station keeping problem of underactuated underwater robot from the robot design perspectives. The proposed scheme is having dedicated small actuators which are used for compensating the external disturbances and at the same time minimizing the power consumption. Since in the underwater mission due to its increasing load of responsibilities minimizing power consumption is necessary in order to maximizing the robot performance in other aspects and moreover in the present situation the robot has to perform multiple missions in a single launch such as inspection, oceanography, intervention tasks, etc $[23,24]$. The proposed scheme is concentrating the station keeping performance of the 
underwater robot under different operating conditions. The wave effects are extensively studied in the literature $[15,25]$. However, the effect of underwater currents is less explored in the previous studies. Therefore, the present work is mainly concentrated on the robot performance under different underwater currents and it also investigates the robot controller adaptability for the robot parameter uncertainties.

The remaining paper is organised in the following manner, the design modification for station keeping is described in the Section 2. Dynamic modelling of robot is discussed in the Section 3. Section 4 contains the brief description of robot controller design. Simulation results and their detailed discussions are given in Section 5 which includes the comparative results of tracking control performance with original AUV design and proposed design, vehicle station keeping performance under different operating conditions (underwater current effects) and uncertainty analysis. Section 6 contains main conclusions of this work along with future formulations and steps for validating the proposed scheme methods.

\section{DESIGN MODIFICATION FOR STATION KEEPING}

An underactuated experimental autonomous underwater robot is considered here for analysis. The robot does not have any side and vertical thrusters to control the sway and heave direction for tracking control mode (these are not implemented because of economical and weight considerations). There are only two stern propellers and stern control planes which provide control inputs for surge, yaw (by differential mode operation of propellers) and pitch motions (refer Fig. 1).

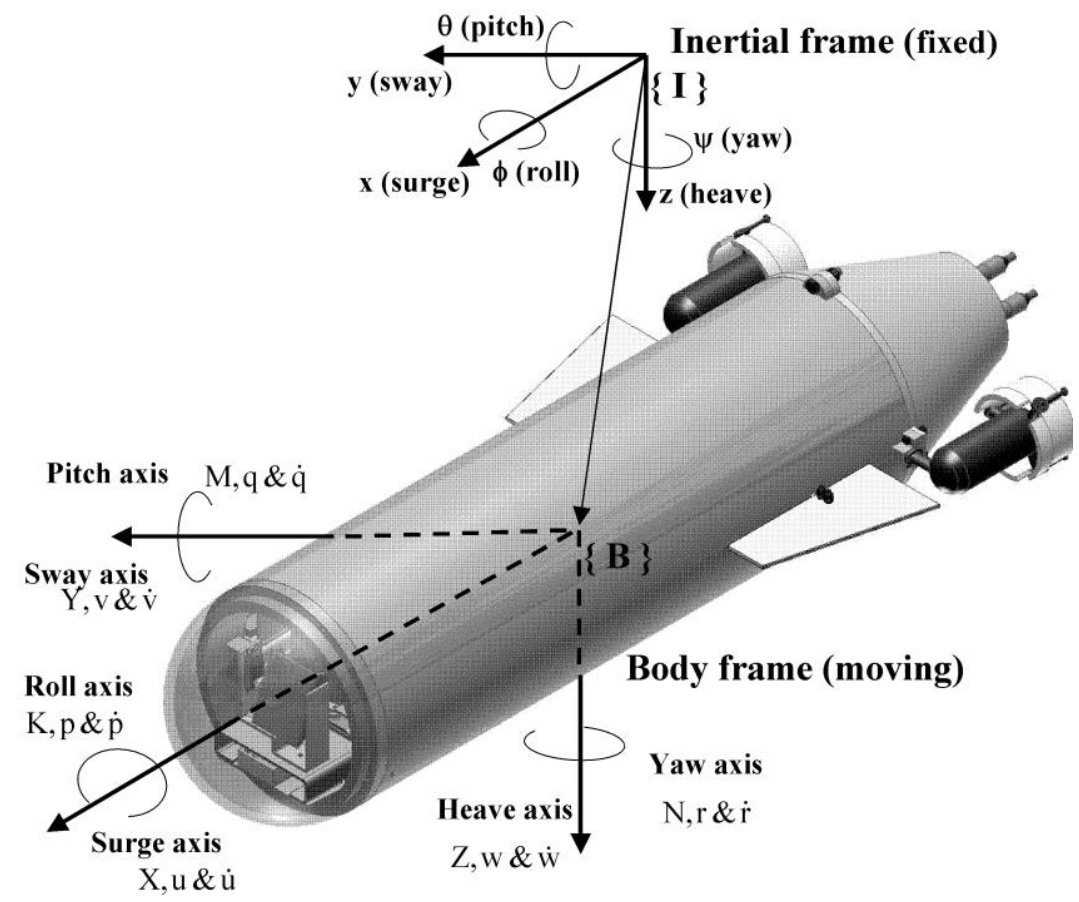

Figure 1: Body-fixed frame and earth-fixed reference frame for underwater robot.

Since the vehicle original actuator configuration is mainly designed to fulfil the tracking control performance, and actuators are consuming high power in order to track the given desired trajectory. The same configuration can not used for station keeping purpose, since the heave can be controlled through the pitch displacement, however the pitch angle is controlled by the help of stern control planes. These stern control plane forces and moments are dependent on the vehicle speed; during station keeping the vehicle speed is nearly zero. 
Similarly in the other case, the sway motion is achieved through yaw angle. Therefore the existing actuator configuration is no longer valid for station keeping control. So, in this work, there are three small additional thrusters are introduced in this robot for station keeping purpose alone, which are less power consuming. The proposed actuator configuration is shown in Fig. 2a. The position and orientation of these thrusters is chosen in such a way that the generated forces and torque are enough to compensate the underwater currents and disturbances during station keeping. Since these parameter values are having large influence in the robot station keeping performance. Therefore design of these values is nearly optimized using Taguchi's robust optimization method.

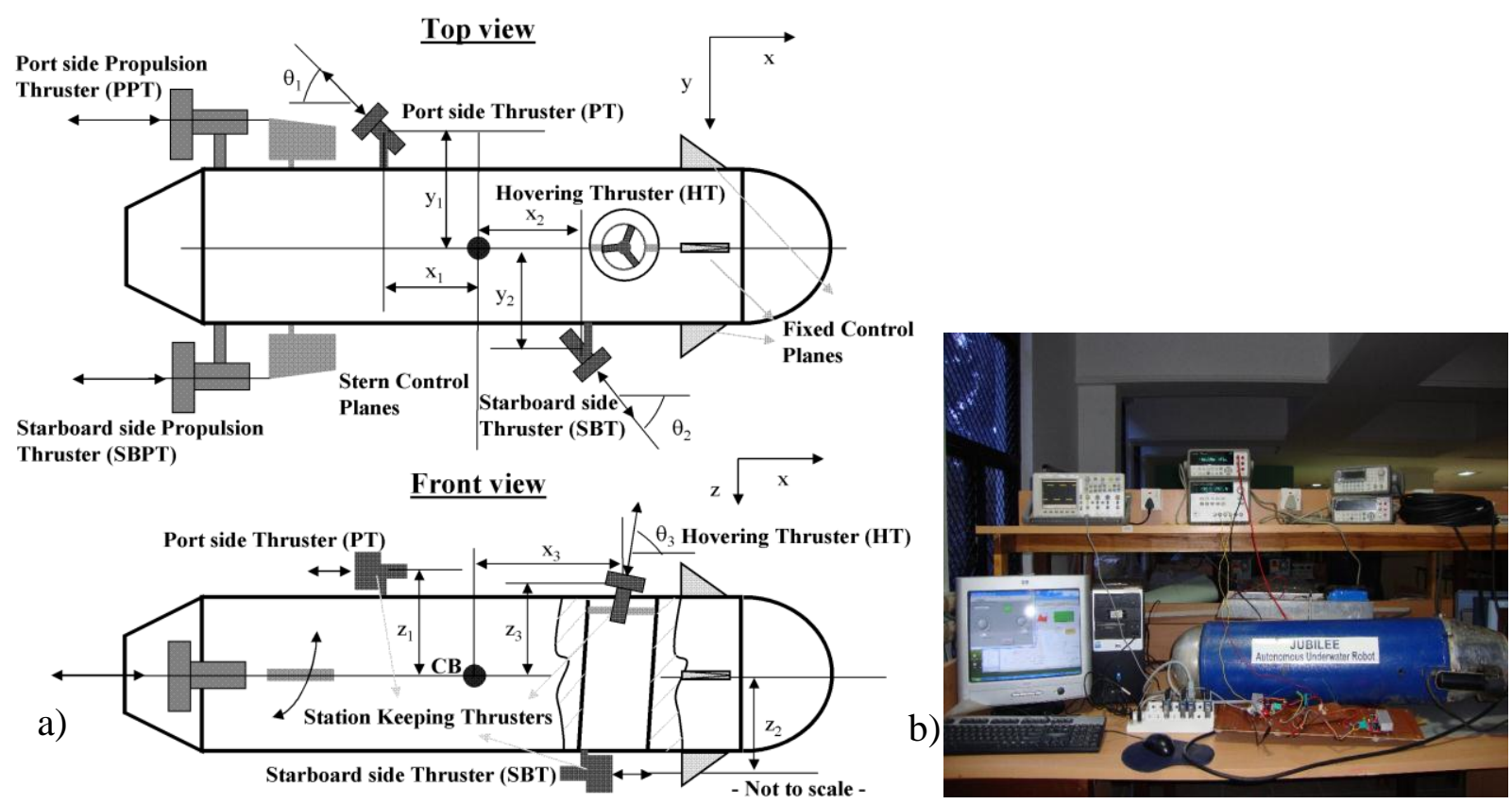

Figure 2: a) Proposed actuators configuration for station keeping;

b) Hardware-in-the-loop simulation setup with Jubilee underwater robot.

\section{DYNAMIC MODELLING OF ROBOT}

Underwater robots experience a range of forces while moving in the fluid medium and they are generally referred to as hydrodynamic forces. A detailed discussion on hydrodynamic forces on underwater robots can be found in [3,4]. Added mass, drag and lift are the most common reaction forces acting on the AUR and these are related to linear and angular accelerations and velocities. The hydrodynamic parameters relate these forces to the corresponding robot parameters that are highly non-linear. The dynamic motion of the underwater robot can be described in a common way using six degrees of freedom (DOF) nonlinear equations (refer Fig. 1). The equations of motion of an underwater robot having six degrees of freedom with respect to a body - fixed frame of reference can be written as [3]:

$$
\mathbf{M} \dot{v}+\mathbf{C}(v) v+\mathbf{D}(v) v+\mathbf{g}(\eta)=\tau
$$

where,

$$
\mathbf{M}=\mathbf{M}_{R B}+\mathbf{M}_{A}, \mathbf{C}(v)=\mathbf{C}_{R B}(v)+\mathbf{C}_{A}(v), \mathbf{D}(v)=\mathbf{D}_{L}+\mathbf{D}_{Q}|v| .
$$

$\mathbf{M}_{R B}$ and $\mathbf{C}_{R B}(v)$ are the rigid body mass matrix and the Coriolis and centripetal matrix, respectively. $\mathbf{M}_{A}$ and $\mathbf{C}_{A}(v)$ are the added mass matrix and the added Coriolis and centripetal matrix respectively. $\mathbf{D}_{L}$ and $\mathbf{D}_{Q}|v|$ are the linear and quadratic drag matrices, respectively. $\mathbf{g}(\eta)$ is the resultant vector of gravity and buoyancy effects. $\tau=\left[\begin{array}{llllll}X & Y & Z & K & M & N\end{array}\right]^{T}$ are the 
resultant forces and moments (which include control inputs such as thruster forces and control plane effects) acting on the robot. $X$ - resultant force in surge direction, $Y$ - resultant force in sway direction, $Z$ - resultant force in heave direction, $K$ - resultant moment in roll axis, $M-$ resultant moment in pitch axis, $N$ - resultant moment in yaw axis.

$\dot{v}=\left[\begin{array}{llllll}\dot{u} & \dot{v} & \dot{w} & \dot{p} & \dot{q} & \dot{r}\end{array}\right]^{T}$ are linear and angular accelerations with respect to body (moving) frame, $\dot{u}, \dot{v}$ and $\dot{w}$ are linear accelerations in surge, sway and heave direction respectively. $\dot{p}, \dot{q}$ and $\dot{r}$ are angular accelerations in roll, pitch and yaw direction respectively. $v=\left[\begin{array}{llllll}u & v & w & p & q & r\end{array}\right]^{T}$ are linear and angular velocities with respect to body (moving) frame, $u$ - surge velocity, $v$ - sway velocity, $w$ - heave velocity, $p$ - roll rate, $q$ - pitch rate, $r$ - yaw rate. $\eta=\left[\begin{array}{lllllll}x & y & z & \varphi & \theta & \psi\end{array}\right]^{T}$ are positions and orientations with respect to inertial (fixed) frame, $x$ - surge position, $y$ - sway position, $z$ - heave position, $\phi$ - roll angle, $\theta$ - pitch angle, $\psi$ - yaw angle.

The relationship between linear and angular velocities in robot frame to those in absolute frame (refer Fig. 1) is given by:

$$
\dot{\eta}=\mathbf{J}(\eta) v
$$

where, $\mathbf{J}(\eta)$ is the kinematic transformation matrix and it is in the following form:

$$
\mathbf{J}(\eta)=\left[\begin{array}{cccccc}
c \psi c \theta & -s \psi c \phi+c \psi s \theta s \phi & s \psi s \phi+c \psi c \theta s \phi & 0 & 0 & 0 \\
s \psi c \theta & c \psi c \phi+s \psi s \theta s \phi & -c \psi s \phi+s \psi s \theta c \phi & 0 & 0 & 0 \\
-s \theta & c \theta s \phi & c \theta c \phi & 0 & 0 & 0 \\
0 & 0 & 0 & 1 & s \phi t \theta & c \phi t \theta \\
0 & 0 & 0 & 0 & c \phi & -s \phi \\
0 & 0 & 0 & 0 & s \phi / c \theta & c \phi / c \theta
\end{array}\right]
$$

where, $s=\sin , c=\cos$ and $t=\tan$ (or $\operatorname{tg}$ ). This transformation is undefined for $\theta= \pm 90^{\circ}$ and to overcome this singularity, a quaternion approach must be considered. However, most of the robots are designed to operate at pitch angles well below $\pm 90^{\circ}$ and hence this limitation has no major significance here.

For the better understanding and good detailed analysis, it is preferred to investigate the system with respect to the earth fixed frame of reference in order to maintain every state to a single reference frame. For this, the coordinate transformation $(\eta, v) \stackrel{\mu}{\rightarrow}(\eta, \dot{\eta})$ is performed using Eq. (3), which yields:

$$
\left(\begin{array}{c}
\eta \\
\dot{\eta}
\end{array}\right)=\left[\begin{array}{cc}
\mathbf{I} & 0 \\
0 & \mathbf{J}(\eta)
\end{array}\right]\left(\begin{array}{l}
\eta \\
v
\end{array}\right)
$$

The coordinate transformation $\mu$ is a global diffeomorphism, analogous to a similarity transformation in linear systems. The robot dynamic model with respect to the earth fixed frame of reference becomes:

$$
\mathbf{M}_{\eta} \ddot{\eta}+\mathbf{C}_{\eta} \dot{\eta}+\mathbf{D}_{\eta} \dot{\eta}+\mathbf{g}_{\eta}=\tau_{\eta}
$$

where,

$$
\begin{aligned}
& \mathbf{M}_{\eta}=\mathbf{J}(\eta)^{-T} \mathbf{M} \mathbf{J}(\eta)^{-1} \\
& \mathbf{C}_{\eta}=\mathbf{J}(\eta)^{-T}\left(\mathbf{C}(v)-\mathbf{M} \mathbf{J}(\eta)^{-1} \mathbf{J}(\eta)\right) \mathbf{J}(\eta)^{-1} \\
& \mathbf{D}_{\eta}=\mathbf{J}(\eta)^{-T} \mathbf{D}(v) \mathbf{J}(\eta)^{-1} \\
& \mathbf{g}_{\eta}=\mathbf{J}(\eta)^{-T} \mathbf{g}(\eta) \\
& \tau_{\eta}=\mathbf{J}(\eta)^{-T} \tau
\end{aligned}
$$

Here, [3] properties of the dynamic system: 


$$
\begin{aligned}
& \mathbf{M}_{\eta}=\mathbf{M}_{\eta}^{T}>0, \forall \eta \in \mathfrak{R}^{6} \\
& \mathbf{s}^{T}\left[\dot{\mathbf{M}}_{\eta}-\mathbf{C}_{\eta}\right] \mathbf{s}=0, \forall \mathbf{s} \in \mathfrak{R}^{6}, v \in \mathfrak{R}^{6}, \eta \in \mathfrak{R}^{6} \\
& \mathbf{D}_{\eta}>0, \forall v \in \mathfrak{R}^{6}, \eta \in \mathfrak{R}^{6}
\end{aligned}
$$

In addition to controller design, the dynamic model of the robot is getting changed because of inclusion of new actuators. In general these thrusters which are small in size, however these damping effects should be considered in the model. Therefore, robot equations of motions (6) are modified as follows:

$$
\mathbf{M}_{s k} \ddot{\eta}+\mathbf{C}_{s k} \dot{\eta}+\mathbf{D}_{s k} \dot{\eta}+\mathbf{g}_{s k}=\tau_{\eta}
$$

where,

$$
\mathbf{M}_{s k}=\mathbf{M}_{\eta}+\mathbf{M}_{s}, \mathbf{C}_{s k}=\mathbf{C}_{\eta}+\mathbf{C}_{s}, \mathbf{D}_{s k}=\mathbf{D}_{\eta}+\mathbf{D}_{s}, \mathbf{g}_{s k}=\mathbf{g}_{\eta}+\mathbf{g}_{s} .
$$

$\mathbf{M}_{s k}, \mathbf{C}_{s k}, \mathbf{D}_{s k}$ and $\mathbf{g}_{s k}$ are the mass matrix , the Coriolis and centripetal matrix, the damping matrix and restoring effects vector which are included the effect of station keeping actuators inclusion, respectively. $\mathbf{M}_{s}, \mathbf{C}_{s}, \mathbf{D}_{s}$ and $\mathbf{g}_{s}$ are the mass matrix, the Coriolis and centripetal matrix, the damping matrix and restoring effects vector which are the effect of station keeping actuators alone, respectively. The inclined water column which presents in the proposed configuration may causes serious problem in the control engineer point of view. However, in this work this particular effect is considered as an added mass which is given in matrix $\mathbf{M}_{s}$ to the robot. Similarly, the portside and starboard side thrusters' and their locations can cause sometime roll instability. For minimizing this effect fixed control surfaces are included. However, these issues are to be addressed in the near future.

In the dynamic model of the robot (due to station keeping actuators inclusion), apart from inertial effects (due to weight and added mass effects of additional thrusters); damping effects are considered as the major effects as compared to other effects and the damping effects of the additional actuators are given by Eq. (11):

where,

$$
\mathbf{D}_{s} \dot{\eta}=\left(\begin{array}{l}
\mathbf{F} \\
\mathbf{T}
\end{array}\right)
$$

$$
\begin{aligned}
& \mathbf{F}_{1}=\left[\begin{array}{ccc}
-c \varphi_{1} & s \varphi_{1} & 0 \\
-s \varphi_{1} & -c \varphi_{1} & 0 \\
0 & 0 & 1
\end{array}\right]\left[\begin{array}{c}
F_{D I} \\
F_{L 1} \\
0
\end{array}\right], \mathbf{F}_{2}=\left[\begin{array}{ccc}
-c \varphi_{2} & s \varphi_{2} & 0 \\
-s \varphi_{2} & -c \varphi_{2} & 0 \\
0 & 0 & 1
\end{array}\right]\left[\begin{array}{c}
F_{D 2} \\
F_{L 2} \\
0
\end{array}\right], \mathbf{F}_{3}=\left[\begin{array}{ccc}
-c \varphi_{3} & 0 & s \varphi_{3} \\
0 & 1 & 0 \\
-s \varphi_{3} & 0 & -c \varphi_{3}
\end{array}\right]\left[\begin{array}{c}
F_{D I} \\
0 \\
F_{L 1}
\end{array}\right], \\
& F_{D}=\frac{1}{2} \rho A C_{D} U^{2}, F_{L}=\frac{1}{2} \rho A C_{L} U^{2} ; C_{D}=0.0002 \varphi_{a}^{2}+0.0012 \varphi_{a}+0.0221 \text {, and } C_{L}=0.0246 \varphi_{a} \\
& \mathbf{F}_{\text {fins }}=\mathbf{F}_{\mathrm{h}_{-} \text {fins }}+\mathbf{F}_{\mathrm{v}_{-} \text {fins }} \\
& \mathbf{F}_{\mathrm{h}_{\text {fins }}}=\left[\begin{array}{c}
F_{D f} \\
0 \\
F_{L f}
\end{array}\right] \text { and } \mathbf{F}_{\mathrm{v}_{-} \text {fins }}=\left[\begin{array}{c}
F_{D f} \\
F_{L f} \\
0
\end{array}\right] \\
& F_{D f}=\rho A_{f} C_{D f} U^{2}, F_{L f}=\rho A_{f} C_{L f} U^{2} ; C_{D f}=0.11 \text { and } C_{L f}=1.1 \\
& \mathbf{T}=\mathbf{r}_{1} \times \mathbf{F}_{1}+\mathbf{r}_{2} \times \mathbf{F}_{2}+\mathbf{r}_{3} \times \mathbf{F}_{3}+\mathbf{T}_{\text {fins }} \\
& \mathbf{T}_{\text {fins }}=\left(\begin{array}{lll}
0 & x_{f} F_{L f} & x_{f} F_{L f}
\end{array}\right)^{\mathrm{T}} \\
& \mathbf{r}_{1}=\left(\begin{array}{lll}
x_{1} & y_{1} & z_{1}
\end{array}\right)^{T}, \mathbf{r}_{2}=\left(\begin{array}{lll}
x_{2} & y_{2} & z_{2}
\end{array}\right)^{T} \text { and } \mathbf{r}_{3}=\left(\begin{array}{lll}
x_{3} & y_{3} & z_{3}
\end{array}\right)^{T}
\end{aligned}
$$

$F_{D}$ and $F_{L}$ are the drag and lift forces which are generated due to the corresponding thrusters' inclusion; $A$ is the approximate contact surface area to the corresponding thrusters. $A_{f}$ is the approximate contact surface area of the fixed control fins. $C_{D}$ and $C_{L}$ are the coefficients of drag and lift. $r$ is the vector of position coordinates. $\varphi_{1}, \varphi_{2}, \varphi_{3}$ are the direction 
of corresponding thrusters axes. $\varphi_{a}$ is the angle of attack which determines the value of coefficients. $x_{f}$ is the distance between fixed control fin centre and robot body centre.

In this work, all the hydrodynamic parameters are estimated using empirical relations based on strip theory. Some parameters like inertia, centre of gravity, centre of buoyancy are calculated from the geometrical design of the vehicle.

\section{CONTROLLER DESIGN}

In order to investigate the effect of the proposed actuator configuration on the performance of the station keeping of underwater robot, the PID controller is simply adopted and in this section, it describes the design of the nonlinear position controller for the AUV to maintain a given reference coordinates using the PID control law.

Let us define a positive definite Lyapunov function as:

$$
V(\tilde{\eta}, \dot{\tilde{\eta}}, \mathbf{w})=\frac{1}{2}\left[\begin{array}{c}
\mathbf{w} \\
\tilde{\eta} \\
\dot{\tilde{\eta}}
\end{array}\right]^{T}\left[\begin{array}{ccc}
\frac{1}{\alpha} \mathbf{K}_{I} & 0 & 0 \\
0 & \alpha \mathbf{K}_{D} & -\alpha \mathbf{M}_{\eta} \\
0 & -\alpha \mathbf{M}_{\eta} & \mathbf{M}_{\eta}
\end{array}\right]\left[\begin{array}{l}
\mathbf{w} \\
\tilde{\eta} \\
\dot{\tilde{\eta}}
\end{array}\right]+\frac{1}{2} \tilde{\eta}^{T}\left[\mathbf{K}_{P}-\frac{1}{\alpha} \mathbf{K}_{I}\right] \tilde{\eta}+U\left(\eta_{d}-\tilde{\eta}\right)-U\left(\eta_{d}\right)+\tilde{\eta}^{T} \mathbf{g}\left(\eta_{d}\right)
$$

Choosing a control input of the form as given below:

$$
\begin{aligned}
& \mathbf{f}=\mathbf{B}^{-1}\left(\mathbf{K}_{P} \tilde{\eta}+\mathbf{K}_{D} \dot{\tilde{\eta}}+\mathbf{K}_{I} \xi\right) \\
& \dot{\xi}=\tilde{\eta}
\end{aligned}
$$

will lead to the vehicle position, orientation and velocity errors tending to zero asymptotically, i.e., the vehicle will maintain the given desired coordinates.

Here, $\mathbf{K}_{P}, \mathbf{K}_{D}$ and $\mathbf{K}_{I}$ are the gain matrices for the proportional, derivative, and integral segments of the controller. These are chosen to be diagonal and positive definite matrices, $\alpha$ is a positive constant, which satisfies $\frac{\lambda_{\min }\left\{\mathbf{M}_{\eta}\right\} \lambda_{\min }\left\{\mathbf{K}_{D}\right\}}{\lambda_{\max }{ }^{2}\left\{\mathbf{M}_{\eta}\right\}}>\alpha>\frac{\lambda_{\max }\left\{\mathbf{K}_{I}\right\}}{\lambda_{\min }\left\{\mathbf{K}_{P}\right\}-k_{g}}, \lambda_{\max }$ and $\lambda_{\min }$ are the maximum and minimum Eigen values respectively, $k_{g}$ is a real constant, $\tilde{\eta}=\eta_{d}-\eta$ denotes the position and orientation errors, $\dot{\tilde{\eta}}=\dot{\eta}_{d}-\dot{\eta}$ denotes the velocity error, $\eta_{d}$ and $\dot{\eta}_{d}$ are the desired values of positions and velocities respectively.

$\mathbf{w}=\tilde{\eta}+\alpha\left(\xi-\mathbf{K}_{I}^{-1} \mathbf{g}\left(\eta_{d}\right)\right)$ is a state vector. $U($.) denotes potential energy of the vector, $\xi$ is the vector of integral error, $\mathbf{B}$ is the input matrix and $\mathbf{f}$ is the control input vector, where,

$$
\begin{aligned}
& \boldsymbol{\tau}_{\eta}=\mathbf{B f}=\text { control inputs, } \\
& \mathbf{B}= \begin{cases}\mathbf{B}_{1} & \text { (for tracking control) } \\
\mathbf{B}_{2} & \text { (for station keeping control) }\end{cases} \\
& \mathbf{f}=\left\{\begin{array}{lll}
{\left[\begin{array}{lll}
T_{1} & T_{2} & \delta s
\end{array}\right]^{T} \quad \text { (for tracking control) }} \\
{\left[\begin{array}{lll}
f_{1} & f_{2} & f_{3}
\end{array}\right]^{T} \quad \text { (for station keeping control) }}
\end{array}\right.
\end{aligned}
$$

$T_{1}$ and $T_{2}$ are the thrust developed by portside and starboard side stern propulsion thrusters respectively. $\delta s$ is the stern control plane angle. $f_{1}, f_{2}$ and $f_{3}$ are the thrust developed by forward, portside and starboard side station keeping thrusters respectively. $\mathrm{B}_{1}$ and $\mathrm{B}_{2}$ are the input matrices of tracking control and station keeping control respectively. These matrices are consists of actuators locations and directions. These controller parameter values are nearly optimized by Taguchi's robust method by running sequence of simulation runs [26]. The stability of the PID controller of the robot can be verified using Lyapunov's direct method and Barbalat's lemma [27, 28]. 


\section{RESULTS AND DISCUSSIONS}

To illustrate the performance of the proposed scheme, typical simulations are conducted and results are presented in this section. For confirming the effectiveness of the proposed actuator configuration, hardware-in-the-loop (HIL) simulation is also carried out, where the control outputs are given as the inputs of the actual actuators and the actuators responses are feedback to the simulation model. For numerical and HIL simulations, Jubilee, the test bed underwater robot (Fig. 2b shows the first prototype of this robot, along with its experimental setup) is considered. Numerical simulations are performed using fourth order Runge-Kutta (rk4) method with a fixed step size of $0.1 \mathrm{~s}$. The actuators and their dynamics are known to influence the overall robot dynamics. Therefore the controller parameters (PID gains) are tuned by considering the actuator characteristics such as actuator response time (time constant, steady state time), limits of actuators (saturation), etc. using Taguchi's robust design method (please refer to [26] for detailed description of this tuning method). Usually, one needs to run a few simulations in order to select the values of the controller gains, locations and angle of directions of the thrusters that give the best compromise in terms of transient behaviour, robustness, and tracking accuracy. Our final choice of controller gain values, thruster positions and orientations obtained from Taguchi's robust design method are given in Table I.

Table I: Vehicle actuator configuration parameters and controller gains.

\begin{tabular}{|c|c|c|}
\hline $\mathbf{K}_{P}=25 \mathbf{I}_{6 \times 6}$ & $\mathbf{K}_{I}=0.5 \mathbf{I}_{6 \times 6}$ & $\mathbf{K}_{D}=50 \mathbf{I}_{6 \times 6}$ \\
\hline$x_{1}=0.2 \mathrm{~m}$ & $x_{2}=-0.25 \mathrm{~m}$ & $x_{3}=0.2 \mathrm{~m}$ \\
\hline$y_{1}=-0.2 \mathrm{~m}$ & $y_{2}=0.25 \mathrm{~m}$ & $y_{3}=0.0 \mathrm{~m}$ \\
\hline$z_{1}=0.2 \mathrm{~m}$ & $z_{2}=-0.25 \mathrm{~m}$ & $z_{3}=-0.15 \mathrm{~m}$ \\
\hline$\varphi_{1}=15^{\circ}$ & $\varphi_{2}=75^{\circ}$ & $\varphi_{3}=75^{\circ}$ \\
\hline
\end{tabular}

\subsection{Effect of station keeping thrusters' inclusion in the existing tracking control}

In order to demonstrate the adaptability of the proposed actuator scheme, the station keeping actuator damping effects are included in the existing tracking control scheme. Desired state trajectories are given in Fig. 3 for the above purpose. The robot accelerates for the first 50 seconds, reaches a constant speed of $1 \mathrm{~m} / \mathrm{s}$ and decelerates in the last 50 seconds. The corresponding desired time trajectories of surge, sway, heave, roll, pitch and yaw for the given 3D trajectory are depicted in Fig. 3.
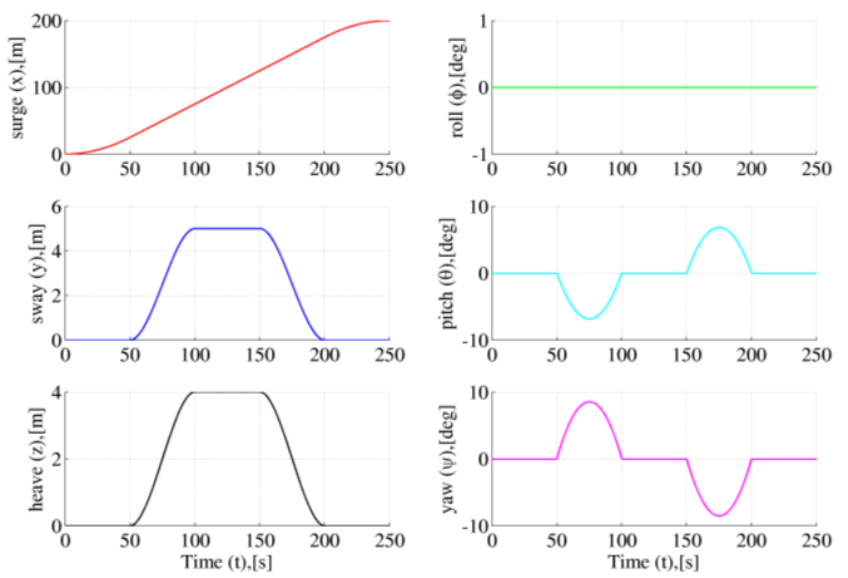

Figure 3: Desired time trajectory of robot states for tracking control. 

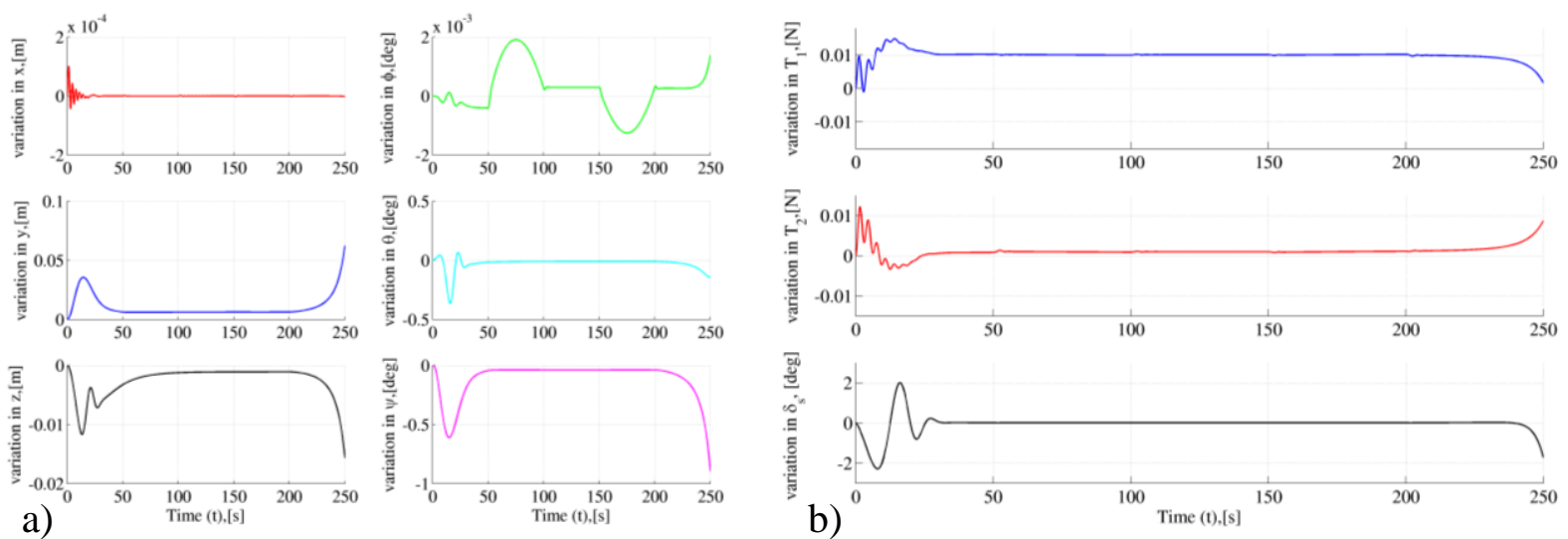

Figure 4: a) Variation in robot states for the inclusion of station keeping actuators;

b) Variation in actuator inputs for the inclusion of station keeping actuators.

The simulation results show that the proposed actuator configuration inclusion is not much influencing (affecting) the existing tracking system performance. The variations of the robot states and control inputs are given in Figs. $4 \mathrm{a}$ and $4 \mathrm{~b}$ respectively. The errors are high during acceleration and deceleration stages i.e., first and last $50 \mathrm{~s}$. Control inputs (see Fig. 4b) which are helping to overcome the inclusion effects of the station keeping thrusters are very minimal (less than $\pm 0.015 \mathrm{~N}$ and $\pm 2.5^{\circ}$ ) and the error variations are less than $10 \mathrm{~mm}$ and $1^{\circ}$ in the positions and attitudes respectively.

\subsection{Simple station keeping results in the presence of underwater current}

The testing condition is chosen as: underwater current amplitude is $0.3 \mathrm{~m} / \mathrm{s}$, angle of attack $45^{\circ}$, side slip angle $45^{\circ}$, and the final desired positions $(x, y$ and $z$ ) and angles (roll, pitch and yaw) are taken as $1 \mathrm{~m}$ and $0^{\circ}$ respectively. Robot desired positions are given as unit step input, apart from this input the underwater current is acting on the robot which tries to drift the robot in its desired states. This case is simulated and results are given in the Fig. 5, which shows that the proposed configuration can compensate the underwater current effects and maintain the robot in its desired states effectively. These results show that the actuator inputs are not exceeding the design limits $( \pm 1.0 \mathrm{kgf}= \pm 9.81 \mathrm{~N})$ and the error trajectories are also converging fast (within 30 to $40 \mathrm{~s}$ ) towards their desired states.
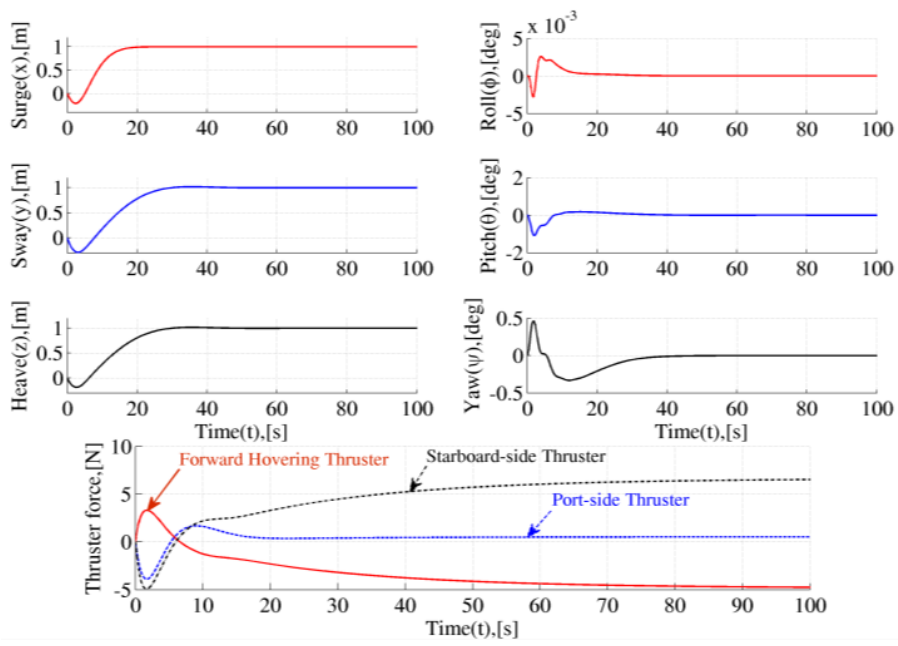

Figure 5: Station keeping results for the proposed configuration with underwater current (condition: $V_{c}=0.3 \mathrm{~m} / \mathrm{s}, \alpha=45^{\circ}$ and $\beta=45^{\circ}$ ). 


\subsection{Hardware-in-the-loop simulation results in the presence of underwater current}

The same previous operating condition has taken for the HIL simulation to confirm the performance of the proposed scheme in the real time. The schematic layout of HIL simulation is presented in Fig. 6. The results of the experiment are given in Fig. 7, which shows that the proposed system can effectively keep the given desired coordinates in the presence of uncertainties such as disturbances (underwater current). The comparative results of HILS (detailed thrusters and control plane dynamics are considered in the simulation model) and numerical simulations (which are given in Sec. 5.2) are given in Figs. 5 and 7, which confirmed that the proposed actuator configuration can compensate the underwater currents effectively. The settling time or steady state time (from HIL values), corresponding to position errors of robot in the surge, sway and heave axes are $29.1 \mathrm{~s}, 23.9 \mathrm{~s}$ and $23.6 \mathrm{~s}$ (within $\pm 5 \mathrm{~cm}$ error limit) respectively, and corresponding to orientation errors in the pitch and yaw axes are $24.7 \mathrm{~s}$ and $28.4 \mathrm{~s}$ (within $\pm 0.3^{\circ}$ error limit). The time history of thrusters outputs are given in Fig. 7, and from this, it is seen that these actuator values are not exceeding the design limits $( \pm 1.0 \mathrm{kgf}= \pm 9.81 \mathrm{~N})$. HIL values are showing similar behaviour of the numerical simulation values with noise effects. These noise effects are mainly due to the actuator response delay, instrument noises and mechanical vibrations of the robot - actuator frame.

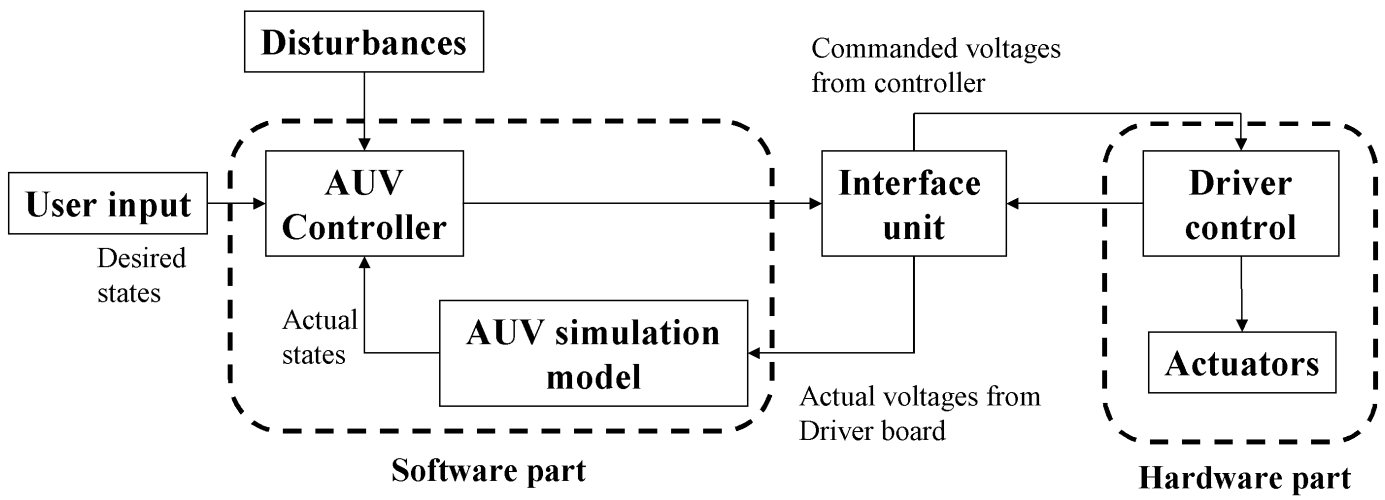

Figure 6: Schematic layout of HILS.
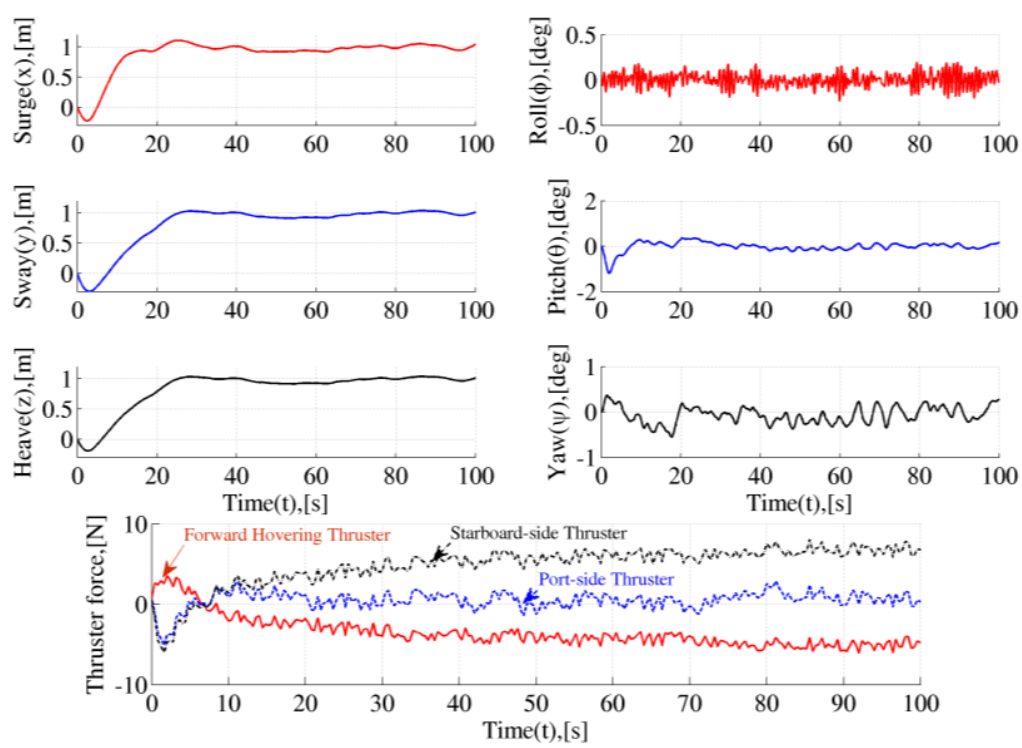

Figure 7: HIL simulation results for the proposed scheme with underwater current (condition: $V_{c}=0.3 \mathrm{~m} / \mathrm{s}, \alpha=45^{\circ}$ and $\beta=45^{\circ}$ ). 


\subsection{Station keeping performance analysis under different underwater currents}

In order to investigate the performance of the proposed configuration in the presence of different underwater currents, there were four parameters have selected such as average current velocity $\left(V_{c}\right)$, angle of attack $(\alpha)$, side slip angle $(\beta)$ and net positive buoyancy $(b)$. The numerical simulations are carried out for current amplitudes varying from $-0.5 \mathrm{~m} / \mathrm{s}$ to $+0.5 \mathrm{~m} / \mathrm{s}$, in steps of $0.1 \mathrm{~m} / \mathrm{s}$, angle of attack and side slip angle are varying from $-90^{\circ}$ to $+90^{\circ}$, in steps of $5^{\circ}$ and the robot net positive buoyancy is varying from $0 \mathrm{kgf}$ to $0.5 \mathrm{kgf}$, in steps of $0.05 \mathrm{kgf}$. The tracking position and attitude errors are plotted in Figs. 8 to 9. These results show that the proposed configuration is producing a much better performance and keeping the robot at the desired coordinates. The maximum position errors, orientation errors and the maximum thrust value are not exceeding $\pm 1 \mathrm{~m}, \pm 2^{\circ}$ and $\pm 6 \mathrm{~N}$ respectively which are within the design limits. The heave position maximum overshoot is almost constant (nearly $1 \mathrm{~m}$ ) for the all the three cases except buoyancy variation plot (Fig. 8). This difference is common phenomenon, since the buoyancy force always tries to push the robot up and the underwater current is also acting in the same direction i.e., the robot getting more effect in the opposite to the desired direction. This comprehensive study helps the design team to identify the possible configuration and modification of the design at the initial stage.
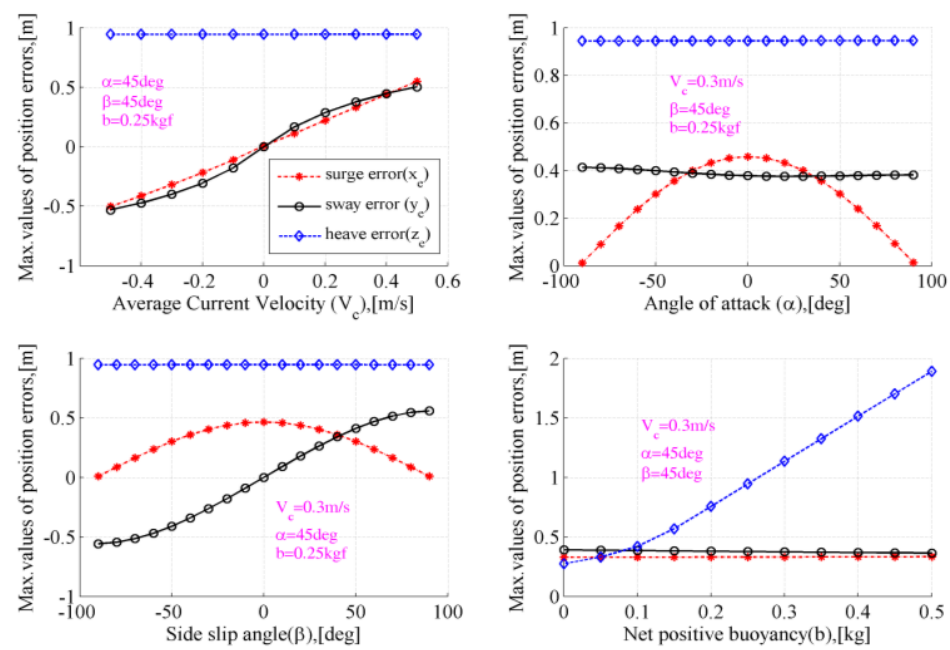

Figure 8: Maximum values of position errors for different underwater currents.
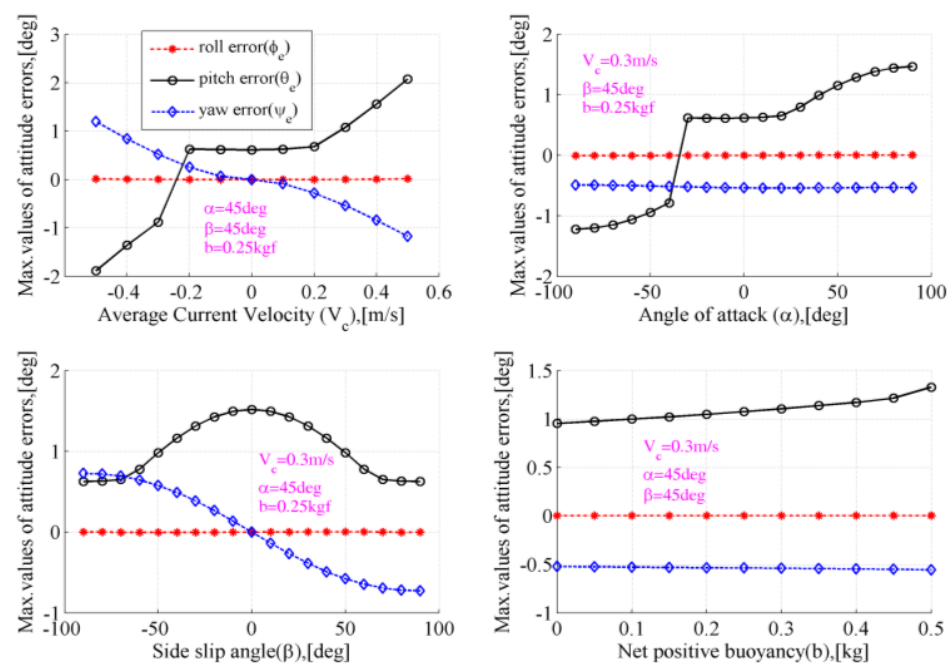

Figure 9: Maximum values of attitude errors for different underwater currents. 


\section{CONCLUSIONS}

In the paper, a series of performance analysis of station keeping control of an autonomous underwater robot has been investigated. The influences and effects of underwater current magnitudes and angle of incidences are analysed.

The simple structure, robustness and ease of computation of the proposed configuration make it very attractive for real time implementation for dynamic positioning of underwater robot. Station keeping actuators inclusion is not much affecting the original tracking control and its performance, and variations (additional effects) in the control inputs are very minimal. Simulation and hardware-in-the-loop simulation results show that the proposed scheme can effectively maintain the given desired position in the presence of underwater currents and uncertainties in hydrodynamic parameters. These simulation results will be very helpful to study the underwater current effect on the underwater robot controller design and its improvement.

The present PID controller is proved to have very good performance to control the underwater robot in the underwater currents, and even good for the parameter uncertainties. However, for the large current amplitudes and buoyancies, the present controller may cause a serious directional instability on the robot; therefore the more robust controller may be needed to improve the present control scheme. Experimental verification of the underwater robot station keeping control will be taken up in the near future to validate the proposed scheme.

\section{REFERENCES}

[1] Blidberg, D. R.; Turner, R. M.; Chappell, S. G. (1991). Autonomous underwater vehicles: Current activities and research opportunities, Robotics and Autonomous Systems, Vol. 7, 139150, doi:10.1016/0921-8890(91)90038-M

[2] Yuh, J. (2000). Design and control of autonomous underwater robots: A survey, Autonomous Robots, Vol. 8, No. 1, 7-24, doi:10.1023/A:1008984701078

[3] Fossen, T. I. (1994). Guidance and Control of Ocean Vehicles, Wiley, Chichester

[4] Sen, D. (2000). A study on sensitivity of maneuverability performance on the hydrodynamic coefficients for submerged bodies, Journal of Ship Research, Vol. 44, No. 3, 186-196

[5] Fossen, T. I. (2002). Marine Control Systems: Guidance, Navigation and Control of Ships, Rigs and Underwater Vehicles, Marine Cybernetics, Trondheim, Norway

[6] Choi, S. K.; Yuh, J. (1996). Experimental Study on a Learning Control System with Bound Estimation for Underwater Robots, Autonomous Robots, Vol. 3, No. 3, 187-194, doi:10.1007/BF00141154

[7] Antonelli, G. (2007). On the use of adaptive / integral actions for six-degrees-of-freedom control of autonomous underwater vehicles, IEEE Journal of Oceanic Engineering, Vol. 32, 300-312, doi:10.1109/JOE.2007.893685

[8] Do, K. D.; Pan, J.; Jiang, Z. P. (2004). Robust and adaptive path following for underactuated autonomous underwater vehicles, Ocean Engineering, Vol. 31, No. 16, 1967-1997, doi:10.1016/j.oceaneng.2004.04.006

[9] Lapierre, L.; Soetanto, D. (2007). Nonlinear path-following control of an AUV, Ocean Engineering, Vol. 34, No. 11-12, 1734-1744, doi:10.1016/j.oceaneng.2006.10.019

[10] Repoulias, F.; Papadopoulos, E. (2007). Planar trajectory planning and tracking control design for underactuated AUVs, Ocean Engineering, Vol. 34, No. 11-12, 1650-1667, doi:10.1016/j.oceaneng.2006.11.007

[11] Kumar, R. P.; Kumar, C. S., Sen, D.; Dasgupta, A. (2009). Discrete time-delay control of an autonomous underwater vehicle: Theory and experimental results, Ocean Engineering, Vol. 36, No.1, 74-81, doi:10.1016/j.oceaneng.2008.07.011 
[12] Perrier, M.; Canudas-De-Wit, C. (1996). Experimental Comparison of PID vs. PID plus Nonlinear Controller for Subsea Robots, Autonomous Robots, Vol. 3, No. 2-3, 195-212, doi:10.1007/BF00141155

[13] Brutzman, D.; Doucy, O.; Healy, A. (2000). Near surface manoeuvring and station-keeping for an autonomous underwater vehicle, Proceedings of the NATO symposium on Applied Vehicle Technology, 1-10

[14] Koh, T. H.; Lau, M. W. S.; Seet, G.; Low, E. (2006). A control module scheme for an underactuated underwater robotic vehicle, Journal of Intelligent and Robotic Systems, Vol. 46, No. 1, 43-58, doi:10.1007/s10846-006-9052-6

[15] Liu, S.; Wang, D.; Poh, E. (2008). Output feedback control design for station keeping of AUVs under shallow water wave disturbances, International Journal of Robust and Nonlinear Control, Vol. 19, No. 13, 1447-1470, doi:10.1002/rnc.1387

[16] Lots, J. F.; Lane, D. M.; Trucco, E.; Chaumette, F. (2001). A 2D visual servoing for underwater vehicle station keeping, Proceedings of the IEEE International Conference on Robotics and Automation, 2767-2772

[17] Riedel, J. S. (2000). Shallow water station keeping of an autonomous underwater vehicle: The experimental results of a disturbance compensation controller, Proceedings of the MTS/IEEE Conference on OCEANS, 1017-1024

[18] Zwaan, S. V.; Bernardino, A.; Santos-Victor, J. (2002). Visual station keeping for floating robots in unstructured environments, Robotics and Autonomous Systems, Vol. 39, 145-155, doi:10.1016/S0921-8890(02)00200-2

[19] Marks, R. L.; Wang, H. H.; Lee, M. J.; Rock, S. M. (1994). Automatic visual station keeping of an underwater robot, Proceedings of the MTS/IEEE Conference on OCEANS, Vol. 2, 137-142

[20] Negahdaripour, S.; Fox, J. (1991). Underwater optical station-keeping: improved methods, Journal of Robotic Systems, Vol. 8, No. 3, 319-338, doi:10.1002/rob.4620080304

[21] Jin, L.; Xu, X.; Negahdaripour, S. (1996). A real-time vision-based stationkeeping system for underwater robotics applications, Proceedings of the MTS/IEEE Conference on OCEANS, Vol. 3 , 1076-1081

[22] Santhakumar, M.; Asokan, T. (2010). Planar dynamic station keeping of underactuated autonomous underwater robot in the presence of underwater currents, Proceedings of the International Symposium on Robotics and Intelligent Sensors, 84-89

[23] Alvarez, A.; Caffaz, A.; Caiti, A.; Casalino, G.; Gualdesi, L.; Turetta, A.; Viviani, R. (2009). Folaga: A low-cost autonomous underwater vehicle combining glider and AUV capabilities, Ocean Engineering, Vol. 36, No. 1, 24-38, doi:10.1016/j.oceaneng.2008.08.014

[24] Chyba, M.; Haberkorn, T.; Singh, S.B.; Smith, R. N.; Choi, S. K. (2009). Increasing underwater vehicle autonomy by reducing energy consumption, Ocean Engineering, Vol. 36, No. 1, 62-73, doi:10.1016/j.oceaneng.2008.07.012

[25] Fang, M. C.; Chang, P. E.; Luo, J. H. (2006). Wave effects on ascending and descending motions of the autonomous underwater vehicle, Ocean Engineering, Vol. 33, 1972-1999, doi:10.1016/j.oceaneng.2005.09.009

[26] Santhakumar, M.; Asokan, T. (2009). Application of robust design techniques for underwater vehicle control, Proceedings of ISOPE Ocean Mining Symposium, 285-289

[27] Slotine, J. E.; Li, W. (1991). Applied Nonlinear Control, Prentice Hall, Inc., Englewood Cliffs

[28] Santhakumar, M.; Asokan T. (2010). Investigations on the hybrid tracking control of an underactuated autonomous underwater robot, Advanced Robotics, Vol. 24, No. 11, 1529-1556, doi:10.1163/016918610X512587 